\title{
Review of: "Precise in vivo functional analysis of DNA variants with base editing using ACEofBASEs target prediction"
}

Daisuke Miki

Potential competing interests: The author(s) declared that no potential competing interests exist.

In the submitted manuscript, Cornean and co-authors established an online prediction tool ACEofBASEs for adenine and cytosine base editing efficiency in medaka and zebrafish. Further, the authors validated the base editing in dapk3, ube2b, usp44, and ptpn11 genes. Overall, their results are interesting and will contribute a lot to the base editing application in fish. In the meantime, there are few aspects that fall short as detailed below, these need to be addressed.

The base editing efficiency depends on the position of the target residues, especially cytosine (Fig. 2e, f, 4d). However, ACEofBASEs predicts the editing efficiency as sgRNA score, not for each target nucleotides. Further, the sgRNA score is represented by the gradient of color. I would like to suggest to show the predicted editing efficiency for each residues as digital score.

The authors used only one sgRNA for each target genes except for kcnh6a. I am wondering how accurate the prediction of editing efficiency by ACEofBASEs is. It would be useful to summarize the predicted and actual editing efficiency for all of the attempted sgRNAs. 